MATHEMATICS OF COMPUTATION

Volume 81, Number 280, October 2012, Pages 2319-2333

S 0025-5718(2011)02566-4

Article electronically published on November 29, 2011

\title{
RIEMANN-SIEGEL INTEGRAL FORMULA FOR THE LERCH ZETA FUNCTION
}

\author{
EUGENIO P. BALANZARIO AND JORGE SÁNCHEZ-ORTIZ
}

\begin{abstract}
Here we present a Riemann-Siegel integral formula for the Lerch zeta function. Proceeding as in Turing's method for computing the Riemann zeta function, our integral formula allows for the numerical computation of the Lerch zeta function by numerical quadratures.
\end{abstract}

\section{INTRODUCTION}

The Lerch zeta function is an important generalization of the Riemann zeta function. Some references on the basic properties of the Lerch zeta function are [10], 9] and [8]. On the other hand, in 1943, A.M. Turing [12] devised a method of computing the Riemann zeta function $\zeta(s)$. See also [6]. Turing's method rests upon the Riemann-Siegel integral formula (see section 7.9 in [5]) which is a representation of $\zeta(s)$ in terms of two definite integrals. Here we obtain the following representation of the Lerch zeta function akin to the Riemann-Siegel integral formula for $\zeta(s)$.

Theorem 1. Let $0<a \leq 1$, let $0<\lambda<1$, and let

$$
L(\lambda, a, s)=\sum_{n=0}^{\infty} \frac{e^{2 \pi i \lambda n}}{(n+a)^{s}} \quad \text { for } s=\sigma+i \text { such that } \sigma>1 .
$$

For the analytic continuation of $L(\lambda, a, s)$ to the half-plane $\sigma>0$, we have

$$
\begin{aligned}
L(\lambda, a, s)= & e^{-i \pi a(1+a+2 \lambda)} \int_{0 \searrow a} \frac{e^{-i \pi w^{2}+2 \pi i w(a+\lambda)}}{e^{-2 \pi i a} e^{i \pi w}-e^{-i \pi w}} w^{-s} d w \\
& +i e^{\pi i \lambda(\lambda+1)+\frac{\pi}{2} i s} \frac{\Gamma(1-s)}{(2 \pi)^{1-s}} \int_{\mathcal{H}} \frac{e^{i \pi w^{2}+2 \pi i w(a+\lambda)}}{e^{2 \pi i \lambda} e^{i \pi w}-e^{-i \pi w}} w^{s-1} d w .
\end{aligned}
$$

Here $\mathcal{H}$ is the Hankel contour which goes from the point at infinity $\infty e^{\frac{\pi}{4} i}$ down to the origin $w=0$, then it goes in the negative sense along a small circle with center $w=0$ and finally goes back to the point at infinity $\infty e^{\frac{\pi}{4} i} . B y 0 \searrow a$ is meant the contour with parametrization $w=\alpha+\xi e^{-\frac{\pi}{4} i}$, where $\alpha \in(0, a)$ is fixed and $\xi$ goes from $-\infty$ to $\infty$.

With this representation for $L(\lambda, a, s)$ it is possible to proceed as in Turing's method and compute $L(\lambda, a, s)$ by numerical quadrature. In order to do so, it is convenient to shift the contour $0 \searrow a$ to the right and to dilate the small circle in the Hankel contour $\mathcal{H}$. The purpose of performing these two processes of shifting and dilating is that the new contours of integration passes through saddle points.

Received by the editor September 27, 2010 and, in revised form, April 15, 2011.

2010 Mathematics Subject Classification. Mathematics subject classification Primary 11M35.

Key words and phrases. Lerch zeta function, Riemann-Siegel, saddle point. 
Once the contours of integration goes through saddle points, the resulting integrals can be computed by a suitable numerical scheme, Simpson's rule for example. In $\S 3$ below we set the stage for the numerical computation of the Lerch zeta function by quadratures. More precisely, we reduce the computation of $L(\lambda, a, s)$ to the numerical calculation of two integrals, a task easily done by an electronic computer.

Let us remark that a Riemann-Siegel integral formula for Dirichlet $L$-series is already known in the literature; see for example 2. The present authors took special care to ensure that the numerical evaluation of the Lerch zeta function based on Theorem 1 were successfully accomplished. It is interesting to note that Theorem 1 holds only for $0<\lambda<1$ and hence an integral representation of the Hurwitz zeta function (corresponding to $\lambda \in \mathbf{Z}$ ) is not a particular case of Theorem 1.

For convenience of the reader, let us recall that when $x, \sigma$ and $t$ are real numbers such that $x>0$ and $s=\sigma+i t$, then $x^{s}$ stands for $x^{\sigma} e^{i t \log x}$.

\section{Proof of Theorem 1}

Our starting point is the following lemma (see [1] for the proof).

Lemma 2. Let $0<a \leq 1$. Let $\beta$ be a real number such that

$$
\frac{\beta}{2}-a+1<1<\frac{\beta}{2}-a+2
$$

Let

$$
\Phi_{a}(\beta)=\frac{1}{2 \pi i} \int_{L} \frac{e^{\frac{i}{4 \pi} u^{2}+\beta u}}{e^{-2 \pi i a} e^{u}-1} d u
$$

where $L$ is the contour with parametrization $u=i \pi \beta+\xi e^{\frac{5}{4} \pi i}$ and $-\infty<\xi<\infty$. Then we have

$$
\Phi_{a}(\beta)=\frac{e^{i \pi\left\{\frac{\beta^{2}}{2}+\beta(a-1)-\frac{a^{2}}{2}+a-\frac{5}{8}\right\}}}{\cos \pi(\beta-a+1)} \cos \frac{\pi}{2}\left(\beta-a+\frac{\sqrt{5}}{2}\right)\left(\beta-a-\frac{\sqrt{5}}{2}\right) .
$$

For the proof of Theorem 1 we need to consider the following two integrals $I(s)$ and $J(s)$. Let us first write

$$
f(w, \lambda)=\frac{e^{-i \pi w^{2}+2 \pi i w(a-\lambda)}}{e^{-2 \pi i a} e^{i \pi w}-e^{-i \pi w}} \quad \text { and } \quad h(w, \lambda)=\frac{e^{i \pi w^{2}+2 \pi i w(a-\lambda)}}{e^{-2 \pi i \lambda} e^{i \pi w}-e^{-i \pi w}} .
$$

For $s=\sigma+i t$ such that $\sigma<1$ let

$$
I(s)=\int_{0 \nwarrow a} f(w, \lambda) w^{s-1} d w \quad \text { and } \quad J(s)=-2 i \sin (\pi s) e^{i \pi s} \int_{0}^{\infty i^{1 / 2}} h(w, \lambda) w^{-s} d w
$$

where $0 \nwarrow a$ is the contour with parametrization $\alpha+\xi e^{\frac{3}{4} \pi i}$ with $-\infty<\xi<\infty$ and $\alpha \in(0, a)$ is fixed. Moreover, the integral $J(s)$ is taken along the contour $w=\xi e^{\frac{\pi}{4} i}$ with $0 \leq \xi<\infty$. 
Proposition 5. Let $0<a \leq 1$ and $0<|\lambda|<1$. Let $s=\sigma+i$ be such that $\sigma<1$. Then

$$
L(-\lambda, a, 1-s)=-e^{-i \pi a(1+a-2 \lambda)} I(s)-e^{i \pi \lambda(\lambda-1)-\frac{\pi}{2} i s} \frac{\Gamma(s)}{(2 \pi)^{s}} J(s)
$$

where $I(s)$ and $J(s)$ are as in (4).

Proof. From Lemma 2 we have

$$
\begin{aligned}
& \frac{1}{2 \pi i} \int_{L} \frac{e^{\frac{i}{4 \pi} u^{2}+\beta u}}{e^{-2 \pi i a} e^{u}-1} d u \\
& \quad=\frac{e^{i \pi\left\{\frac{\beta^{2}}{2}+\beta(a-1)-\frac{a^{2}}{2}+a-\frac{5}{8}\right\}}}{\cos \pi(\beta-a+1)} \cos \frac{\pi}{2}\left(\beta-a+\frac{\sqrt{5}}{2}\right)\left(\beta-a-\frac{\sqrt{5}}{2}\right)
\end{aligned}
$$

where $L$ is the contour $u=i \pi \beta+\xi e^{\frac{5}{4} \pi i}$ and $-\infty<\xi<\infty$. Therefore

(6) $\frac{1}{2 \pi i} \int_{L} \frac{e^{\frac{i}{4 \pi} u^{2}+\beta u}}{e^{-2 \pi i a} e^{u}-1} d u=\frac{e^{i \pi \beta^{2}+i \pi(a-\beta)-\frac{5}{4} \pi i}}{e^{i \pi(\beta-a+1)}+e^{-i \pi(\beta-a+1)}}-\frac{e^{-i \pi a^{2}+2 \pi i a \beta+2 \pi i(a-\beta)}}{1+e^{-2 \pi i(\beta-a+1)}}$.

Writing $u=2 \pi i w$ we obtain

$$
\frac{1}{2 \pi i} \int_{L} \frac{e^{\frac{i}{4 \pi} u^{2}+\beta u}}{e^{-2 \pi i a} e^{u}-1} d u=\int_{(a-1) \nwarrow a} \frac{e^{-i \pi w^{2}+2 \pi i \beta w}}{e^{-2 \pi i a} e^{2 \pi i w}-1} d w
$$

where the relation between $a$ and $\beta$ in Lemma 2 ensures that the path $L$ is transformed into $(a-1) \nwarrow a$. Taking $\beta=v+1 / 2$ we see that (6) and (7) imply

$$
I_{1}(v)=I_{2}(v)+I_{3}(v)
$$

where

$$
\begin{gathered}
I_{1}(v)=\int_{(a-1) \nwarrow a} \frac{e^{-i \pi w^{2}+2 \pi i v w+i \pi w}}{e^{-2 \pi i a} e^{2 \pi i w}-1} d w, \quad I_{2}(v)=-\frac{e^{i \pi v^{2}+i \pi a}}{e^{i \pi(v-a)}-e^{-i \pi(v-a)}}, \\
I_{3}(v)=\frac{e^{-i \pi a^{2}+3 \pi i a-2 \pi i v+2 \pi i v a}}{1-e^{-2 \pi i(v-a)}} .
\end{gathered}
$$

Let us now multiply both sides of (8) by $(v-a+\lambda)^{-s}$ where $0<|\lambda|<1$ and $\sigma<1$, and then let us integrate with respect to $v$ along the path $v=a-\lambda+\xi e^{\frac{\pi}{4} i}$ where 
$0 \leq \xi<\infty$. Then we have

$$
\begin{gathered}
\int_{a-\lambda}^{\infty i^{1 / 2}} I_{3}(v)(v-a+\lambda)^{-s} d v=\int_{a-\lambda}^{\infty i^{1 / 2}} \frac{e^{i \pi\left(3 a-a^{2}\right)+2 \pi i v(a-1)}}{1-e^{-2 \pi i(v-a)}}(v-a+\lambda)^{-s} d v \\
=e^{i \pi\left(a^{2}+a\right)} \int_{0}^{\infty i^{1 / 2}} \frac{e^{2 \pi i(a u+\lambda-u-a \lambda)}}{1-e^{-2 \pi i(u-\lambda)} u^{-s} d u} \\
=e^{i \pi\left(a^{2}+a\right)-2 \pi i \lambda a} \int_{0}^{\infty i^{1 / 2}} \frac{e^{-2 \pi i(u-\lambda)}}{1-e^{-2 \pi i(u-\lambda)}} e^{2 \pi i u a} u^{-s} d u \\
=-e^{i \pi\left(a^{2}+a\right)-2 \pi i \lambda a} \int_{0}^{\infty i^{1 / 2}} e^{2 \pi i u a} \sum_{n=0}^{\infty} e^{2 \pi i n(u-\lambda)} u^{-s} d u \\
=-e^{i \pi\left(a^{2}+a\right)-2 \pi i \lambda a} \sum_{n=0}^{\infty} e^{-2 \pi i n \lambda} \int_{0}^{\infty i^{1 / 2}} e^{2 \pi i u(n+a)} u^{-s} d u \\
=e^{i \pi\left(a^{2}+a\right)-2 \pi i \lambda a} \frac{i e^{\frac{i \pi}{2} s}}{(2 \pi)^{1-s}} \sum_{n=0}^{\infty} \frac{e^{-2 \pi i n \lambda}}{(n+a)^{1-s}} \int_{0}^{\infty i^{3 / 2}} e^{w} w^{-s} d w \\
=-i e^{i \pi\left(a^{2}+a\right)-2 \pi i \lambda a} e^{-\frac{\pi i}{2} s} \frac{\Gamma(1-s)}{(2 \pi)^{1-s}} \sum_{n=0}^{\infty} \frac{e^{-2 \pi i n \lambda}}{(n+a)^{1-s}} .
\end{gathered}
$$

If $|\lambda| \in(0,1)$, then $I_{3}(v)$ does not have a singularity at $v=a-\lambda$. Hence the integral $\int_{a-\lambda}^{\infty i^{1 / 2}} I_{3}(v)(v-a+\lambda)^{-s} d v$ converges uniformly in $s$ on each compact subset of $\sigma<1$. Therefore (for $\sigma<1$ ),

$$
\int_{a-\lambda}^{\infty i^{1 / 2}} \frac{I_{3}(v)}{(v-a+\lambda)^{s}} d v=-i e^{i \pi a(a+1-2 \lambda)-\frac{\pi i}{2} s} \frac{\Gamma(1-s)}{(2 \pi)^{1-s}} L(-\lambda, a, 1-s) .
$$

We write this briefly as

$$
\int I_{3}=\mathrm{X}_{3} L \quad \text { where } \quad \mathrm{X}_{3}=-i e^{i \pi a(a+1-2 \lambda)-\frac{\pi i}{2} s} \frac{\Gamma(1-s)}{(2 \pi)^{1-s}} .
$$

On the other hand,

$$
\begin{gathered}
\int_{a-\lambda}^{\infty i^{1 / 2}} I_{2}(v)(v-a+\lambda)^{-s} d v=\int_{a-\lambda}^{\infty i^{1 / 2}} \frac{e^{i \pi v^{2}+i \pi a}}{e^{-i \pi(v-a)}-e^{i \pi(v-a)}}(v-a+\lambda)^{-s} d v \\
=-\int_{0}^{\infty i^{1 / 2}} \frac{e^{i \pi\left(v^{2}+a^{2}+\lambda^{2}\right)-2 \pi i \lambda(v+a)+i \pi a(2 v+1)}}{e^{i \pi(v-\lambda)}-e^{-i \pi(v-\lambda)}} v^{-s} d v \\
=-e^{i \pi a(a+1)+i \pi \lambda(\lambda-2 a)} \int_{0}^{\infty i^{1 / 2}} \frac{e^{i \pi v^{2}+2 \pi i v(a-\lambda)}}{e^{i \pi(v-\lambda)}-e^{-i \pi(v-\lambda)}} v^{-s} d v .
\end{gathered}
$$


We write this briefly as

$$
\int I_{2}=\mathrm{X}_{2} \int_{0}^{\infty i^{1 / 2}} \quad \text { where } \quad \mathrm{X}_{2}=-e^{i \pi a(a+1)+i \pi \lambda(\lambda-2 a)} .
$$

Now we consider the integral

$$
\int_{a-\lambda}^{\infty i^{1 / 2}} \frac{I_{1}(v)}{(v-a+\lambda)^{s}} d v=\int_{a-\lambda}^{\infty i^{1 / 2}} \int_{(a-1) \nwarrow a} \frac{e^{-i \pi w^{2}+2 \pi i w v+i \pi w}}{e^{-2 \pi i a} e^{2 \pi i w}-1} \cdot \frac{d w d v}{(v-a+\lambda)^{s}} .
$$

Interchanging integrals we obtain

$$
\begin{aligned}
& \int_{a-\lambda}^{\infty i^{1 / 2}} \int_{(a-1) \nwarrow a} \frac{e^{-i \pi w^{2}+2 \pi i w v+i \pi w}}{e^{-2 \pi i a} e^{2 \pi i w}-1}(v-a+\lambda)^{-s} d w d v \\
= & \int_{(a-1) \nwarrow a} \frac{e^{-i \pi w^{2}}}{e^{-2 \pi i a} e^{i \pi w}-e^{-i \pi w}} \int_{a-\lambda}^{\infty i^{1 / 2}} e^{2 \pi i w v}(v-a+\lambda)^{-s} d v d w \\
= & \int_{(a-1) \nwarrow a} \frac{e^{-i \pi w^{2}}}{e^{-2 \pi i a} e^{i \pi w}-e^{-i \pi w}} \int_{0}^{\infty i^{1 / 2}} e^{2 \pi i w(v-\lambda+a)} v^{-s} d v d w \\
= & \int_{(a-1) \nwarrow a} \frac{e^{-i \pi w^{2}+2 \pi i w(a-\lambda)}}{e^{-2 \pi i a} e^{i \pi w}-e^{-i \pi w}} \int_{0}^{\infty i^{1 / 2}} e^{2 \pi i w v} v^{-s} d v d w .
\end{aligned}
$$

In order to justify the exchange of integrals, notice that $(a-1) \nwarrow a$ has $w=$ $\alpha+\xi(-1+i)$ as a parametric representation, where $\alpha$ is a fixed number such that $a-1<\alpha<a$ and $-\infty<\xi<\infty$. On the other hand, we can write $v=\eta(1+i)$ where $0 \leq \eta<\infty$. Now

$$
\Re \mathrm{e}(2 \pi i w v)=-2 \pi \eta \alpha .
$$

Therefore, the integral inside converges absolutely when $\alpha>0$. Henceforth, we will assume $0<\alpha<a$ and we will write $0 \nwarrow a$ instead of $(a-1) \nwarrow a$.

If we write $v=x / 2 \pi i w$, then we have

$$
\int_{0}^{\infty i^{1 / 2}} e^{2 \pi i w v} v^{-s} d v=(2 \pi w)^{s-1} e^{\frac{\pi}{2} i(s-1)} \int_{0}^{\infty e^{i \theta}} e^{x} x^{-s} d x
$$

where $\pi / 2<\theta<3 \pi / 2$. On the other hand,

$$
\int_{0}^{\infty e^{i \theta}} e^{x} x^{-s} d x=\int_{0}^{-\infty} e^{x} x^{-s} d x=-e^{-\pi i s} \Gamma(1-s) .
$$

Therefore

$$
\int_{0}^{\infty i^{1 / 2}} e^{2 \pi i w v} v^{-s} d v=i(2 \pi w)^{s-1} e^{-\frac{\pi}{2} i s} \Gamma(1-s) .
$$

Hence, the double integral in (11) equals

$$
i \Gamma(1-s)(2 \pi)^{s-1} e^{-\frac{\pi}{2} i s} \int_{0 \nwarrow a} \frac{e^{-i \pi w^{2}+2 \pi i w(a-\lambda)}}{e^{-2 \pi i a} e^{i \pi w}-e^{-i \pi w}} w^{s-1} d w .
$$

We write this briefly as

$$
\int I_{1}=\mathrm{X}_{1} \int_{0 \nwarrow a} \quad \text { where } \quad \mathrm{X}_{1}=i \Gamma(1-s)(2 \pi)^{s-1} e^{-\frac{\pi}{2} i s} .
$$


If we take into account (8), (9), (10) and (12), then we find

$$
L=\frac{\mathrm{X}_{1}}{\mathrm{X}_{3}} \int_{0 \nwarrow a}-\frac{\mathrm{X}_{2}}{\mathrm{X}_{3}} \int_{0}^{\infty i^{1 / 2}}
$$

where

$$
\frac{\mathrm{X}_{1}}{\mathrm{X}_{3}}=-\exp \{-i \pi a(a+1-2 \lambda)\}
$$

and where

$$
\frac{\mathrm{X}_{2}}{\mathrm{X}_{3}}=-2 i e^{i \pi \lambda^{2}+\frac{\pi i}{2} s} \frac{\sin (\pi s)}{(2 \pi)^{s}} \Gamma(s) .
$$

This finishes the proof of Proposition 5.

The aim of the following lemma is to change the contour in $J(s)$ into a Hankel contour.

Lemma 13. Let $|\lambda| \in(0,1)$ and $0<r<|\lambda|$. Let $\sigma<1$. Consider the contour of integration $\mathcal{H}(r)=C_{1}(r)+C_{0}(r)+C_{2}(r)$ where $C_{1}, C_{0}$ and $C_{2}$ are as follows:

$$
-C_{1}=\left\{\begin{array}{l}
w=\xi e^{\frac{\pi}{4} i}, \\
r \leq \xi<\infty,
\end{array} \quad-C_{0}=\left\{\begin{array}{l}
w=r e^{\theta i}, \\
-\frac{7 \pi}{4} \leq \theta \leq \frac{\pi}{4},
\end{array} \quad C_{2}=\left\{\begin{array}{c}
w=\xi e^{-\frac{7 \pi}{4} i} \\
r \leq \xi<\infty
\end{array}\right.\right.\right.
$$

Let $J(s)$ be as in (4). Then we have

$$
J(s)=\lim _{r \rightarrow 0} \int_{\mathcal{H}(r)} h(w, \lambda) \frac{d w}{w^{s}} .
$$

Proof. Notice that $h(w, \lambda)$ has poles at $w=k+\lambda$ for $k \in \mathbf{Z}$. Since $|\lambda| \in(0,1)$ and $0 \leq r<|\lambda|$, then $h(w, \lambda)$ is analytic in $|w| \leq r$. Thus, there exists $K$ such that $|h(w, \lambda)| \leq K$ for each $|w| \leq r$. Since $\sigma<1$, then we have

$$
\int_{C_{0}(r)} h(w, \lambda) \frac{d w}{w^{s}} \ll 2 \pi r \cdot K \frac{e^{\frac{\pi}{4} t}}{r^{\sigma}} \rightarrow 0
$$

as $r \rightarrow 0$. For each $0 \leq r<|\lambda|$ we have

$$
\int_{C_{2}(r)} h(w, \lambda) \frac{d w}{w^{s}}=-e^{2 \pi i s} \int_{C_{1}(r)} h(w, \lambda) \frac{d w}{w^{s}} .
$$

Therefore

$$
\int_{\mathcal{H}(0)}=\int_{C_{1}(0)}+\int_{C_{2}(0)}=\left(1-e^{2 \pi i s}\right) \int_{C_{1}(0)}
$$

and hence

$$
\lim _{r \rightarrow 0} \int_{\mathcal{H}(r)} h(w, \lambda) \frac{d w}{w^{s}}=e^{\pi i s}\left(e^{-\pi i s}-e^{\pi i s}\right) \int_{0}^{\infty i^{1 / 2}} h(w, \lambda) \frac{d w}{w^{s}}=J(s) .
$$

Theorem 1 follows at once from Proposition 5 and Lemma 13 after the substitutions $\lambda \mapsto-\lambda$ and $s \mapsto 1-s$ are made. 


\section{Numerical COMPUTATION}

In order to numerically compute the Lerch zeta function, we will first shift the contour $0 \nwarrow a$ in $I(s)$ to the right.

Lemma 14. Let $n_{0} \in \mathbf{N}$ and let $f(w, \lambda)$ be as in (3). Then we have

$$
I(s)=-\sum_{k=0}^{n_{0}} \frac{e^{-2 \pi i \lambda(k+a)+i \pi a(a+1)}}{(k+a)^{1-s}}+\int f(w, \lambda) w^{s-1} d w
$$

where the integral is over $\left(n_{0}+a\right) \nwarrow\left(n_{0}+a+1\right)$.

Proof. Notice that $f(w, \lambda) w^{s-1}$ has poles at $w=k+a$ for each $k \in \mathbf{Z}$, with residues

$$
e^{-2 \pi i \lambda(k+a)+i \pi a(a+1)} \frac{(k+a)^{s-1}}{2 \pi i} .
$$

Since $n_{0}>0$, then we cross each pole by closing a contour with a negative orientation. Therefore the residues must be multiplied by -1 .

We now dilate the small circle in the Hankel contour $\mathcal{H}(r)$ depicted in Lemma 13.

Lemma 15. Let $0<\lambda<1$, let $n_{1} \in \mathbf{N}$ and let $\mathcal{H}(r)$ be as in Lemma 13. Then we have (notice $J(s)=J(s,-\lambda)$ in the expression (4) for $J(s)$ )

$$
\begin{aligned}
J(s)=-e^{\pi i s} & \sum_{k=0}^{n_{1}-1} \frac{e^{-2 \pi i a(k+\lambda)-i \pi \lambda(\lambda+1)}}{(k+\lambda)^{s}} \\
& -\sum_{k=1}^{n_{1}} \frac{e^{2 \pi i a(k-\lambda)-i \pi \lambda(\lambda+1)}}{(k-\lambda)^{s}}+\int_{\mathcal{H}\left(n_{1}\right)} h(w,-\lambda) \frac{d w}{w^{s}} .
\end{aligned}
$$

Proof. If $|\lambda| \in(0,1)$, then $h(w, \lambda) w^{-s}$ has poles at $w=k+\lambda$ for each $k \in \mathbf{Z}$. If $k<0$, then the corresponding residue equals

$$
\frac{e^{2 \pi i a(k+\lambda)-i \pi \lambda(\lambda-1)}}{2 \pi i}|k+\lambda|^{-s} e^{i \pi s} .
$$

If $k \geq 0$, then the corresponding residue equals

$$
\frac{e^{2 \pi i a(k+\lambda)-i \pi \lambda(\lambda-1)}}{2 \pi i}|k+\lambda|^{-s} .
$$

The lemma follows when we add these residues and when we write $-\lambda$ instead of $\lambda$ where $0<\lambda<1$.

Next we exchange the Hankel contour $\mathcal{H}\left(n_{1}\right)$ for two parallel straight lines.

Lemma 16. Let $0<\lambda<1$. Let $n_{1} \in \mathbf{N}$. Let $C_{1}$ be the straight line $w=$ $\alpha_{1}+\xi e^{-\frac{3}{4} \pi i}$ where $\alpha_{1}$ is fixed and such that $n_{1}-\lambda<\alpha_{1}<n_{1}-\lambda+1$ and $-\infty<\xi<\infty$. Let $C_{2}$ be the straight line $w=\alpha_{2}+\xi e^{-\frac{7}{4} \pi i}$ where $\alpha_{2}$ is fixed and such that $-\left(n_{1}+\lambda\right)<\alpha_{2}<-\left(n_{1}+\lambda\right)+1$ and $-\infty<\xi<\infty$. Then we have

$$
\int_{\mathcal{H}\left(n_{1}\right)} h(w,-\lambda) \frac{d w}{w^{s}}=\int_{C_{1}} h(w,-\lambda) \frac{d w}{w^{s}}+\int_{C_{2}} h(w,-\lambda) \frac{d w}{w^{s}} .
$$


Proof. Let $\alpha$ be the intersection point of $C_{1}$ with the real axis. Let $K>0$. We consider the following two paths of integration:

$$
-C_{1}(K)=\left\{\begin{array}{c}
w=\alpha e^{-\frac{\pi}{2} i}+\xi e^{\frac{\pi}{4} i}, \\
-K \leq \xi<\infty,
\end{array} \quad C_{2}(K)=\left\{\begin{array}{c}
w=\alpha e^{-i \pi}+\xi e^{-\frac{7}{4} \pi i}, \\
-K \leq \xi<\infty .
\end{array}\right.\right.
$$

Let $w_{1} \in C_{1}(K)$ and $w_{2} \in C_{2}(K)$ be the points corresponding to $\xi=-K$ in the above representation of $C_{1}(K)$ and $C_{2}(K)$. The integral of $h w^{-s}$ over $\mathcal{H}(\alpha)$ is equal to the integral of $h w^{-s}$ over the path which runs first over $C_{1}(K)$, then runs over the straight line segment joining $w_{1}$ to $w_{2}$ and then runs over $C_{2}(K)$. It is easy to see that the integral over the line segment from $w_{1}$ to $w_{2}$ is

$$
\ll e^{-c_{1} K^{2}} K^{-\sigma}
$$

where $c_{1}>0$. If we let $K \rightarrow \infty$, then we see that the integral over $\mathcal{H}(\alpha)$ equals the integral over $C_{1}(\infty)$ plus the integral over $C_{2}(\infty)$.

In Proposition 17 we make a concise statement of our discussion up to this point. It is this form of the Riemann-Siegel integral formula which will allow us to compute the Lerch zeta function by numerical quadrature. It is easy to shift the path $C_{2}$ in Lemma 16 to the left or to the right as the need arises. When in this shifting process we cross a pole, we must take the corresponding residue into account. Thus we can make the two sums in Lemma 15 have an independent number of terms.

Proposition 17. Let $n_{0}, n_{1}$ and $n_{2}$ be natural numbers. Let $0<\lambda<1$. Let $s=\sigma+i t$ be such that $\sigma<1$. Let $f(w, \lambda)$ and $h(w, \lambda)$ be as in (3). For the Lerch zeta function we have

$$
\begin{gathered}
L(\lambda, a, 1-s)=\sum_{k=0}^{n_{0}} \frac{e^{2 \pi i \lambda k}}{(k+a)^{1-s}}-e^{-i \pi a(1+a+2 \lambda)} \int_{C_{0}} f(w,-\lambda) w^{s-1} d w \\
+e^{-2 \pi i a \lambda} \frac{\Gamma(s)}{(2 \pi)^{s}}\left[e^{\frac{\pi}{2} i s} \sum_{k=0}^{n_{2}-1} \frac{e^{-2 \pi i a k}}{(k+\lambda)^{s}}-e^{-\frac{\pi}{2} i s} \sum_{k=1}^{n_{1}} \frac{e^{2 \pi i a k}}{(k-\lambda)^{s}}\right] \\
-e^{i \pi \lambda(\lambda+1)-\frac{\pi}{2} i s} \frac{\Gamma(s)}{(2 \pi)^{s}}\left\{\int_{C_{1}}+\int_{C_{2}}\right\} h(w,-\lambda) \frac{d w}{w^{s}}
\end{gathered}
$$

where $C_{0}=\left(n_{0}+a\right) \nwarrow\left(n_{0}+a+1\right), C_{1}=\left(n_{1}-\lambda\right) \swarrow\left(n_{1}-\lambda\right)+1$, and finally $C_{2}=-\left(n_{2}+\lambda\right) \nearrow-\left(n_{2}+\lambda\right)+1$.

Now we are ready to apply the saddle point method. For a general description of this method, see [4]. Browsing section 4 of [12] could be beneficial for understanding the application of the saddle point method in the context of a zeta function.

Our task now is to choose $n_{0}, n_{1}$ and $n_{2}$ in Proposition 17 in such a way that the quadrature of the integrals of $f$ and $h$ can be performed conveniently. For the choice of $n_{0}$ notice that $f(w,-\lambda) w^{s-1}$ has two saddle points. By saddle point of $f(w,-\lambda) w^{s-1}$ we mean the value of $w$ such that $\phi^{\prime}(w)=0$, where

$$
\phi(w)=-i \pi w^{2}+2 \pi i w(a+\lambda)+i \pi w+(s-1) \log w .
$$


Hence, the two saddle points of $f(w,-\lambda) w^{s-1}$ are

$$
w=\frac{A}{4} \pm \sqrt{\left(\frac{A}{4}\right)^{2}+\frac{s-1}{2 \pi i}} \quad \text { where } \quad A=2(a+\lambda)+1 .
$$

Notice that the function

$$
f(w,-\lambda)=\frac{e^{-i \pi w^{2}+2 \pi i w(a+\lambda)+i \pi w}}{e^{2 \pi i(w-a)}-1}
$$

has poles at $w=k+a$ for each $k \in \mathbf{Z}$. Now we can choose $n_{0}$. Let

$$
n_{0}=\left[\beta_{0}-a\right] \quad \text { where } \quad \beta_{0}=\frac{A}{4}+\sqrt{\left(\frac{A}{4}\right)^{2}+\frac{t}{2 \pi}} .
$$

In the next lemma we bound the contribution of the integral of $f$ due to the two straight line segments that remain after we remove a central part of the contour $C_{0}$.

Lemma 20. Let $0<\lambda<1$ and $0<a \leq 1$. Let $A=2(a+\lambda)+1$. Let $\beta_{0}$ be as in (19). Let $0<\sigma<1$. For each $K>0$, let

$$
L_{0}(K)=\left\{\begin{array}{c}
w=\beta_{0}+\xi e^{\frac{3 \pi}{4} i}, \\
K \leq|\xi|<\infty .
\end{array}\right.
$$

Let $\Lambda_{0}=44 \pi / 100$. Then we have

$$
\left|\int_{L_{0}(K)} f(w,-\lambda) \frac{d w}{w^{1-s}}\right| \leq \frac{6}{5} \cdot \frac{\beta_{0}^{\sigma-1}}{\Lambda_{0} K} \cdot \frac{e^{-\Lambda_{0} K^{2}}}{\left\|\beta_{0}-a\right\|}
$$

where $\|x\|=|x-[x+1 / 2]|$ is the distance from $x$ to the set $\mathbf{Z}$.

Proof. If $z=x+i y$, then

$$
\left|\frac{1}{e^{2 \pi i z}-1}\right| \leq \max \left\{\frac{6}{5}, \frac{3}{5} \sqrt{\frac{1}{\|x\|^{2}+y^{2}}}\right\} .
$$

Thus, for each $w \in L_{0}(0)$ we have

$$
\left|\frac{1}{e^{2 \pi i(w-a)}-1}\right| \leq \frac{3 \sqrt{2}}{5\left\|\beta_{0}-a\right\|} .
$$

Therefore $\left|\int_{L_{0}(K)} f(w,-\lambda) w^{s-1} d w\right|$ is not greater than

$$
\frac{3 \sqrt{2}}{5|| \beta_{0}-a||} \int_{|\xi| \geq K} \frac{\exp \left\{-\pi \xi^{2}+\sqrt{2} \pi \beta_{0} \xi-\pi A \xi / \sqrt{2}-t F_{0}\left(\xi / \beta_{0}\right)\right\}}{\left|\beta_{0}+\xi e^{\frac{3 \pi}{4}} i\right|^{1-\sigma}} d \xi
$$

where we have written $F_{0}\left(\xi / \beta_{0}\right)$ instead of $\arg \left(\beta_{0}+\xi e^{\frac{3 \pi}{4} i}\right)$. Notice that

$$
F_{0}(\xi)=\left\{\begin{array}{lr}
\arctan \left(\frac{\xi}{\sqrt{2}-\xi}\right) & \text { when } \xi<\sqrt{2}, \\
\pi+\arctan \left(\frac{\xi}{\sqrt{2}-\xi}\right) & \text { when } \xi>\sqrt{2}
\end{array}\right.
$$

Here $\arctan (x)$ denotes the usual arc tangent function, that is, the strictly increasing function such that $\arctan (x) \rightarrow \pm \pi / 2$ when $x \rightarrow \pm \infty$ and such that $\arctan (0)=0$. 
By performing the change of variable $\xi \mapsto \beta_{0} \xi$ we see that $\left|\int_{L_{0}(K)} f(w,-\lambda) w^{s-1} d w\right|$ is not greater than (since $0<\sigma<1$ )

$$
\frac{6 \beta_{0}^{\sigma}}{5|| \beta_{0}-a \|} \int_{|\xi| \geq \frac{K}{\beta_{0}}} \exp \left\{\pi \beta_{0}^{2}\left(-\xi^{2}+\sqrt{2} \xi-\frac{A \xi}{\beta_{0} \sqrt{2}}-\frac{t}{\pi \beta_{0}^{2}} F_{0}(\xi)\right)\right\} d \xi .
$$

On the other hand, for each $t \geq 0$, each $\xi \in \mathbf{R}$ and each $1<A \leq 5$, we have

$$
-\xi^{2}+\sqrt{2} \xi-\frac{A \xi}{\beta_{0} \sqrt{2}}-\frac{t}{\pi \beta_{0}^{2}} F_{0}(\xi) \leq-\frac{44}{100} \xi^{2} .
$$

Therefore $\left|\int_{L_{0}(K)} f(w,-\lambda) w^{s-1} d w\right|$ is not greater than

$$
\frac{12 \beta_{0}^{\sigma}}{5\left\|\beta_{0}-a\right\|} \int_{K / \beta_{0}}^{\infty} e^{-\Lambda_{0}\left(\beta_{0} \xi\right)^{2}} d \xi \quad \text { where } \quad \Lambda_{0}=\frac{44 \pi}{100}=1.3823 \cdots
$$

Finally, notice that

$$
\int_{K / \beta_{0}}^{\infty} e^{-\Lambda_{0}\left(\beta_{0} \xi\right)^{2}} d \xi \leq \frac{e^{-\Lambda_{0} K^{2}}}{2 K \Lambda_{0} \beta_{0}} .
$$

We still have to choose $n_{1}$ and $n_{2}$. Notice that $h(w,-\lambda)$ has two saddle points. By a saddle point of $h(w,-\lambda) w^{-s}$ we mean the value of $w$ such that $\phi^{\prime}(w)=0$, where

$$
\phi(w)=i \pi w^{2}+2 \pi i w(a+\lambda)+i \pi w-s \log w .
$$

Hence, the two saddle points of $h(w,-\lambda) w^{-s}$ are

$$
w=-\frac{A}{4} \pm \sqrt{\left(\frac{A}{4}\right)^{2}+\frac{s}{2 \pi i}} \quad \text { where } \quad A=2(a+\lambda)+1 .
$$

Notice that the function

$$
h(w,-\lambda)=\frac{e^{i \pi w^{2}+2 \pi i w(a+\lambda)+i \pi w}}{e^{2 \pi i(w+\lambda)}-1}
$$

has poles at $w=k-\lambda$ for each $k \in \mathbf{Z}$. Now we can choose $n_{1}$ and $n_{2}$. Let

$$
n_{1}=\left[\beta_{1}+\lambda\right] \quad \text { where } \quad \beta_{1}=-\frac{A}{4}+\sqrt{\left(\frac{A}{4}\right)^{2}+\frac{t}{2 \pi}} .
$$

Finally, let

$$
n_{2}=-\left[\beta_{2}+\lambda\right] \quad \text { where } \quad \beta_{2}=-\frac{A}{4}-\sqrt{\left(\frac{A}{4}\right)^{2}+\frac{t}{2 \pi}} .
$$

In the next lemma we will bound the contribution of the integral of $h$ due to the two straight line segments that remain after we remove a central part of the contour $C_{1}$. We will also consider the integral of $h$ along $C_{2}$.

Lemma 25. Let $0<\lambda<1$ and $0<a \leq 1$. Let $A=2(a+\lambda)+1$. Let $\beta_{1}$ be as in (23). Let $0<\sigma<1$. For each $K>0$, let

$$
L_{1}(K)=\left\{\begin{array}{c}
w=\beta_{1}+\xi e^{-\frac{3 \pi}{4} i} \\
K \leq|\xi|<\infty
\end{array}\right.
$$

Let $\Lambda_{1}=8 \pi / 100$. If $s=\sigma+i t$ is such that $t \geq 7$, then

$$
\left|\int_{L_{1}(K)} h(w,-\lambda) \frac{d w}{w^{s}}\right| \leq \frac{6}{5} \cdot \frac{\beta_{1}^{-\sigma}}{\Lambda_{1} K} \cdot \frac{e^{-\Lambda_{1} K^{2}}}{\left\|\beta_{1}+\lambda\right\|}
$$


where $\|x\|$ is the distance from $x$ to the set $\mathbf{Z}$.

Proof. Because of (21), for each $w \in L_{1}(0)$ we have

$$
\left|\frac{1}{e^{2 \pi i(w+\lambda)}-1}\right| \leq \frac{3 \sqrt{2}}{5\left\|\beta_{1}+\lambda\right\|} .
$$

Therefore $\left|\int_{L_{1}(K)} h(w,-\lambda) w^{-s} d w\right|$ is not greater than

$$
\frac{3 \sqrt{2}}{5\left\|\beta_{1}+\lambda\right\|} \int_{|\xi| \geq K} \frac{\exp \left\{-\pi \xi^{2}+\sqrt{2} \pi \beta_{1} \xi+\pi A \xi / \sqrt{2}+t F_{1}\left(\xi / \beta_{1}\right)\right\}}{\left|\beta_{1}+\xi e^{-\frac{3 \pi}{4} i}\right|^{\sigma}} d \xi
$$

where we have written $F_{1}\left(\xi / \beta_{1}\right)$ instead of $\arg \left(\beta_{1}+\xi e^{-\frac{3 \pi}{4} i}\right)$. Notice that

$$
F_{1}(\xi)= \begin{cases}\arctan \left(\frac{\xi}{\xi-\sqrt{2}}\right) & \text { when } \xi<\sqrt{2}, \\ -\pi+\arctan \left(\frac{\xi}{\xi-\sqrt{2}}\right) & \text { when } \xi>\sqrt{2} .\end{cases}
$$

By performing the change of variable $\xi \mapsto \beta_{1} \xi$ we see that $\left|\int_{L_{1}(K)} h(w,-\lambda) w^{-s} d w\right|$ is not greater than (since $0<\sigma<1$ )

$$
\frac{6 \beta_{1}^{1-\sigma}}{5|| \beta_{1}+\lambda||} \int_{|\xi| \geq \frac{K}{\beta_{1}}} \exp \left\{\pi \beta_{1}^{2}\left(-\xi^{2}+\sqrt{2} \xi+\frac{A \xi}{\beta_{1} \sqrt{2}}+\frac{t}{\pi \beta_{1}^{2}} F_{1}(\xi)\right)\right\} d \xi .
$$

On the other hand, for each $t \geq 7$, each $\xi \in \mathbf{R}$ and each $1<A \leq 5$, we have

$$
-\xi^{2}+\sqrt{2} \xi+\frac{A \xi}{\beta_{1} \sqrt{2}}+\frac{t}{\pi \beta_{1}^{2}} F_{1}(\xi) \leq-\frac{8}{100} \xi^{2} .
$$

Therefore $\left|\int_{L_{1}(K)} h(w,-\lambda) w^{-s} d w\right|$ is not greater than

$$
\frac{12 \beta_{1}^{1-\sigma}}{5\left\|\beta_{1}+\lambda\right\|} \int_{K / \beta_{1}}^{\infty} e^{-\Lambda_{1}\left(\beta_{1} \xi\right)^{2}} d \xi \quad \text { where } \quad \Lambda_{1}=\frac{8 \pi}{100}=0.2513 \cdots
$$

Finally, notice that

$$
\int_{K / \beta_{1}}^{\infty} e^{-\Lambda_{1}\left(\beta_{1} \xi\right)^{2}} d \xi \leq \frac{e^{-\Lambda_{1} K^{2}}}{2 K \Lambda_{1} \beta_{1}} .
$$

For the integral of $h$ along $C_{2}$ we next give two bounds. Notice, in particular, that one of these bounds does not involve the expression $\left\|\beta_{2}+\lambda\right\|$ in the denominator. This is because we have made that $C_{2}$ crosses the real axis as far as it is possible from the poles.

Lemma 26. Let $0<\lambda<1$ and $0<a \leq 1$. Let $A=2(a+\lambda)+1$. Let $0<\sigma<1$. Let $\beta \in\left(-\left(n_{2}+\lambda\right),-\left(n_{2}+\lambda\right)+1\right)$ where $n_{2}$ is as in $(24)$. For each $K>0$, let

$$
L_{2}(K)=\left\{\begin{array}{c}
w=\beta+\xi e^{-\frac{7 \pi}{4} i}, \\
K \leq|\xi|<\infty .
\end{array}\right.
$$

Let $\Lambda_{2}=44 \pi / 100$. If $\beta=\beta_{2}$ where $\beta_{2}$ is as in (24), then

$$
\left|\int_{L_{2}(K)} h(w,-\lambda) \frac{d w}{w^{s}}\right| \leq \frac{6}{5} \cdot \frac{\left|\beta_{2}\right|^{-\sigma}}{\Lambda_{2} K} \cdot \frac{e^{-\Lambda_{2} K^{2}}}{\left\|\beta_{2}+\lambda\right\|} \exp \{-\pi t\} .
$$


If $\beta=-\left(n_{2}+\lambda\right)+\frac{1}{2}$, where $n_{2}$ is as in $(24)$, then

$$
\left|\int_{L_{2}(0)} h(w,-\lambda) \frac{d w}{w^{s}}\right| \leq 4 \exp \left\{17 \pi-\frac{54 \pi}{100} t\right\} .
$$

Proof. Assume $\beta \in\left(-\left(n_{2}+\lambda\right),-\left(n_{2}+\lambda\right)+1\right)$. Because of (21), for each $w \in L_{2}(0)$ we have

$$
\left|\frac{1}{e^{2 \pi i(w+\lambda)}-1}\right| \leq \frac{3 \sqrt{2}}{5\|\beta+\lambda\|} .
$$

Therefore $\left|\int_{L_{2}(K)} h(w,-\lambda) w^{-s} d w\right|$ is not greater than

$$
\frac{3 \sqrt{2}}{5 \| \beta+\lambda||} \int_{|\xi| \geq K} \frac{\exp \left\{-\pi \xi^{2}-\sqrt{2} \pi \beta \xi-\pi A \xi / \sqrt{2}+t F_{2}(\xi /|\beta|)\right\}}{\left|\beta+\xi e^{-\frac{7 \pi}{4} i}\right|^{\sigma}} d \xi
$$

where we have written $F_{2}(\xi /|\beta|)$ instead of $\arg \left(\beta+\xi e^{-\frac{7 \pi}{4} i}\right)$. Notice that

$$
F_{2}(\xi)=\left\{\begin{array}{lc}
-\pi-\arctan \left(\frac{\xi}{\sqrt{2}-\xi}\right) & \text { when } \xi<\sqrt{2} \\
-2 \pi-\arctan \left(\frac{\xi}{\sqrt{2}-\xi}\right) & \text { when } \xi>\sqrt{2}
\end{array}\right.
$$

By performing the change of variable $\xi \mapsto|\beta| \xi$ we see that $\left|\int_{L_{2}(K)} h(w,-\lambda) w^{-s} d w\right|$ is not greater than (since $0<\sigma<1$ )

$$
\frac{6|\beta|^{1-\sigma}}{5|| \beta+\lambda||} \int_{|\xi| \geq \frac{K}{|\beta|}} \exp \left\{\pi \beta^{2}\left(-\xi^{2}+\sqrt{2} \xi+\frac{A \xi}{\beta \sqrt{2}}+\frac{t}{\pi \beta^{2}} F_{2}(\xi)\right)\right\} d \xi .
$$

If $\beta=\beta_{2}$ where $\beta_{2}$ is as in (24), then

$$
-\xi^{2}+\sqrt{2} \xi+\frac{A \xi}{\beta \sqrt{2}}+\frac{t}{\pi \beta^{2}} F_{2}(\xi) \leq-\frac{44}{100} \xi^{2}-\frac{t}{\beta^{2}}
$$

for each $t \geq 0$, each $\xi \in \mathbf{R}$ and each $1<A \leq 5$. Hence $\left|\int_{L_{2}(K)} h(w,-\lambda) w^{-s} d w\right|$ is not greater than

$$
e^{-\pi t} \frac{12\left|\beta_{2}\right|^{1-\sigma}}{5\left\|\beta_{2}+\lambda\right\|} \int_{K /\left|\beta_{2}\right|}^{\infty} e^{-\Lambda_{2}\left(\beta_{2} \xi\right)^{2}} d \xi \quad \text { where } \quad \Lambda_{2}=\frac{44 \pi}{100}=1.3823 \cdots
$$

Finally, notice that

$$
\int_{K /\left|\beta_{2}\right|}^{\infty} e^{-\Lambda_{2}\left(\beta_{2} \xi\right)^{2}} d \xi \leq \frac{e^{-\Lambda_{2} K^{2}}}{2 K \Lambda_{2}\left|\beta_{2}\right|} .
$$

Now assume that $\beta=-\left(n_{2}+\lambda\right)+1 / 2$ where $n_{2}$ is as in (24). In this case, for each $w \in L_{2}(0)$ we have

$$
\left|\frac{1}{e^{2 \pi i(w+\lambda)}-1}\right| \leq \sqrt{2}
$$

Hence $\left|\int_{L_{2}(0)} h(w,-\lambda) w^{-s} d w\right|$ is not greater than

$$
2|\beta|^{1-\sigma} \int_{-\infty}^{+\infty} \exp \left\{\pi \beta^{2}\left(-\xi^{2}+\sqrt{2} \xi+\frac{A \xi}{\beta \sqrt{2}}+\frac{t}{\pi \beta^{2}} F_{2}(\xi)\right)\right\} d \xi .
$$

Since $-7 \pi / 4 \leq F_{2}(\xi) \leq-3 \pi / 4$, then we have

$$
-\xi^{2}+\sqrt{2} \xi+\frac{A \xi}{\beta \sqrt{2}}+\frac{t}{\pi \beta^{2}} F_{2}(\xi) \leq-(\xi-B)^{2}+B^{2}-\frac{3 t}{4 \beta^{2}}
$$


where we have written $B=\frac{1}{\sqrt{2}}+\frac{A}{2 \beta \sqrt{2}}$. Therefore

$$
\beta^{2}\left(B^{2}-\frac{3 t}{4 \beta^{2}}\right)=\left(\frac{\beta}{\sqrt{2}}+\frac{A}{2 \sqrt{2}}\right)^{2}-\frac{3 t}{4} .
$$

Now notice that

$$
\beta=\left[\beta_{2}+\lambda\right]-\lambda+\frac{1}{2} \geq \beta_{2}-\frac{1}{2} \quad \text { so that } \quad|\beta| \leq\left|\beta_{2}\right|+\frac{1}{2} .
$$

Since in addition $A \leq 5$, then

$$
\left|\frac{\beta}{\sqrt{2}}+\frac{A}{2 \sqrt{2}}\right| \leq \frac{\left|\beta_{2}\right|}{\sqrt{2}}+\frac{1}{2 \sqrt{2}}+\frac{5}{2 \sqrt{2}}=\frac{\left|\beta_{2}\right|}{\sqrt{2}}+\frac{3}{\sqrt{2}} .
$$

On the other hand, for each $t \geq 0$,

$$
\left(\left|\beta_{2}\right|+3\right)^{2} \leq\left\{3+\frac{5}{4}+\sqrt{\left(\frac{5}{4}\right)^{2}+\frac{t}{2 \pi}}\right\}^{2} \leq 34+\frac{126}{100} \frac{t}{\pi} .
$$

Thus we see that

$$
\beta^{2}\left(B^{2}-\frac{3 t}{4 \beta^{2}}\right) \leq 17+\frac{63}{100} \frac{t}{\pi}-\frac{3 t}{4} \leq 17-\frac{54}{100} t .
$$

Multiplying this inequality by $\pi$ we see that (27) is not greater than

$$
2|\beta|^{1-\sigma} \exp \left\{17 \pi-\frac{54 \pi}{100} t\right\} \int_{-\infty}^{+\infty} e^{-\pi \beta^{2}(\xi-B)^{2}} d \xi=\frac{2}{|\beta|^{\sigma}} \exp \left\{17 \pi-\frac{54 \pi}{100} t\right\} .
$$

This finishes the proof.

Before we can state a scheme for the numerical calculation of the Lerch zeta function, two more lemmas are in order.

Lemma 28. Let $n_{2}$ be as in (24). Let $0<\lambda<1$. Let $s=\sigma+i t$ be such that $0<\sigma<1$. Then we have

$$
\left|\sum_{k=0}^{n_{2}-1} \frac{e^{-2 \pi i a k}}{(k+\lambda)^{s}}\right| \leq \frac{1}{\lambda^{\sigma}}+\frac{1+\sqrt{t}}{1-\sigma} .
$$

Proof. If $n \geq 0$, then

$$
\sum_{k=0}^{n-1} \frac{1}{(k+\lambda)^{\sigma}} \leq \frac{1}{\lambda^{\sigma}}+\int_{0}^{n} \frac{d x}{(x+\lambda)^{\sigma}} \leq \frac{1}{\lambda^{\sigma}}+\frac{n}{1-\sigma} .
$$

The lemma follows since $n_{2} \leq 1+\sqrt{t}$.

Lemma 29. For the gamma function, we have, for $t \geq 0$,

$$
|\Gamma(\sigma+i t)| \leq \frac{5}{2} \frac{e^{-\frac{\pi}{2} t}}{\sigma}(t+1)^{\sigma-\frac{1}{2}}
$$

Now we can make a brief statement of our discussion up to this point and conclude by giving a scheme for the numerical calculation of the Lerch zeta function $L(\lambda, a, s)$. To this end, let $\gamma_{j}(K)$ be the finite straight line segment such that $C_{j}=\gamma_{j}(K)+L_{j}(K)$ for each $K>0$, where $C_{j}$, with $j=0,1,2$, are as in Proposition 17 and also $L_{0}(K), L_{1}(K)$ and $L_{2}(K)$ are as in Lemmas 20,25 and 26. Thus, $\gamma_{j}(K)$ are the central parts of the paths $C_{j}$, that is, what remains after we remove the extreme parts $L_{j}(K)$. 
If we approximate the Lerch zeta function $L(\lambda, a, 1-s)$ by the terms

$$
\begin{gathered}
\sum_{k=0}^{n_{0}} \frac{e^{2 \pi i \lambda k}}{(k+a)^{1-s}}-e^{-2 \pi i a \lambda-\frac{\pi}{2} i s} \frac{\Gamma(s)}{(2 \pi)^{s}} \sum_{k=1}^{n_{1}} \frac{e^{2 \pi i a k}}{(k-\lambda)^{s}} \\
-e^{-i \pi a(1+a+2 \lambda)} \int_{\gamma_{0}\left(K_{0}\right)} f(w,-\lambda) \frac{d w}{w^{1-s}}-e^{i \pi \lambda(\lambda+1)-\frac{\pi}{2} i s} \frac{\Gamma(s)}{(2 \pi)^{s}} \int_{\gamma_{1}\left(K_{1}\right)} h(w,-\lambda) \frac{d w}{w^{s}},
\end{gathered}
$$

then, for each $t \geq 7$, the resulting error is not greater than

$$
\begin{aligned}
& \frac{6}{5}\left\{\frac{\beta_{0}^{\sigma-1}}{\Lambda_{0} K_{0}} \cdot \frac{e^{-\Lambda_{0} K_{0}^{2}}}{\left\|\beta_{0}-a\right\|}+\frac{5(t+1)^{\sigma-\frac{1}{2}}}{2 \sigma(2 \pi)^{\sigma}} \cdot \frac{\beta_{1}^{-\sigma}}{\Lambda_{1} K_{1}} \cdot \frac{e^{-\Lambda_{1} K_{1}^{2}}}{\left\|\beta_{1}+\lambda\right\|}\right\} \\
+ & \frac{5(t+1)^{\sigma-\frac{1}{2}}}{2 \sigma(2 \pi)^{\sigma}}\left\{4 \exp \left(17 \pi-\frac{54 \pi}{100} t\right)+\left(\frac{1}{\lambda^{\sigma}}+\frac{1+\sqrt{t}}{1-\sigma}\right) e^{-\pi t}\right\}
\end{aligned}
$$

where $\beta_{0}$ and $\beta_{1}$ are as in (19) and (23), respectively.

In the preceding paragraph we expressed the very basic idea of Turing's method of calculation applied to the Lerch zeta function. In Turing's paper [12, as well as in [6], bounds are given for the errors that are incurred, when one approximates the integrals $\int_{\gamma_{j}\left(K_{j}\right)}$ by using Riemann sums. These bounds for the errors are discussed also by Crouch and Spiegelman [3, by Goodwin [7] and by McNamee [11. It is clear, though, that there exist more efficient methods for the numerical calculation of definite integrals, than the method of using Riemann sums, Simpson's rule, for example.

Finally, we call the attention of the reader to the fact that we have approximated the Lerch zeta function in the $L(\lambda, a, 1-s)$ instead of the more natural form $L(\lambda, a, s)$. By approximating $L(\lambda, a, 1-s)$ instead of $L(\lambda, a, s)$ we ensure that the saddle points are very near the real axis. Thus we avoid some exponentially large terms.

\section{REFERENCES}

[1] Balanzario, E.P., A Riemann-Siegel formula for the Hurwitz zeta function., Bol. Soc. Mat. Mexicana (3) 10 (2004), no. 1, 1-13. MR2071998 (2005d:11132)

[2] Deuring, M., Asymptotische Entwicklungen der Dirichletschen L-Reihen., Math. Ann. 168, 1967, 1-30. MR0213309 (35:4173)

[3] Crouch, E.A.C.; Spiegelman, D., The evaluation of integrals of the form $\int_{-\infty}^{+\infty} f(t) \exp \left(-t^{2}\right) d t$, application to logistic-normal models, J. Amer. Statist. Assoc. 85 (1990), no. 410, 464-469. MR.1141749 (92h:65032)

[4] de Bruijn, N.G., Asymptotic methods in analysis, North-Holland, 1958. MR0099564(20:6003)

[5] Edwards, H.M., Riemann's zeta function, Pure and Applied Mathematics, Vol. 58. Academic Press, New York-London, 1974. MR.0466039 (57:5922)

[6] Galway, W.F., Computing the Riemann zeta function by numerical quadrature, Dynamical, spectral, and arithmetic zeta functions, San Antonio, TX, 1999, 81-91, Contemp. Math., 290, Amer. Math. Soc., 2001. MR.1868470(2002i:11131)

[7] Goodwin, E.T., The evaluation of integrals of the form $\int_{-\infty}^{\infty} f(x) e^{-x^{2}} d x$., Proc. Cambridge Philos. Soc. 45 (1949), 241-245. MR0029281(10:575f)

[8] Lagarias, J.C.; Wen-Ching, Winnie Li, The Lerch zeta function I. Zeta integrals, Forum Mathematicum, published online.

[9] Laurinčikas, A.; Garunkštis, R., The Lerch zeta-function, Kluwer, 2002. MR:1979048 (2004c:11161)

[10] Lerch, M., Note sur la fonction $\mathcal{K}(w, x, s)=\sum_{k=0}^{\infty} e^{2 k \pi i x}(w+k)^{-s}$, Acta Math. 11 (1887), 19-24. MR 1554747 
[11] McNamee, J., Error-bounds for the evaluation of integrals by the Euler-Maclaurin formula and by Gauss-type formulae, Math. Comp. 18 (1964), 368-381. MR0185804 (32:3264)

[12] Turing, A.M., A method for the calculation of the zeta-function, Proc. London Math. Soc. (2) 48 (1943), 180-197. MR0009612 (5:173a)

Instituto de Matemáticas, Unidad Morelia, Universidad Nacional Autónoma de México E-mail address: ebg@matmor.unam.mx

Apartado Postal 61-3 (Xangari), 58089, Morelia Michoacán, México Current address: Facultad de Matemáticas, Av. Lázaro Cárdenas S/N, Ciudad Universitaria, Chilpancingo Gro., México

E-mail address: jsanchez@matmor.unam.mx 